\title{
Meta-analytic Re-assessment of the Validity of Miller Forensic Assessment Test for Detection of Malingering
}

\author{
Zack Z. Cernovsky
}

\section{ABSTRACT}

Background: The Miller Forensic Assessment of Symptoms Test (M-FAST) is used widely for the assessment of malingering of medical symptoms. Its validity has allegedly been supported by meta-analytic study of M-FAST in 2019 by Detullio et al. Credibility of Detullio's results is damaged by an inclusion of data based on analog validation and also on dubious convergent validation procedures that falsify estimates of M-FAST's validity.

Method: In the present study, the meta-analysis was calculated on 3 types of M-FAST data: (1) 5 samples of scores of healthy persons instructed to respond honestly, (2) 5 samples of scores of medical patients, and (3) 10 samples of scores of healthy persons instructed to feign mental illness (so called "instructed malingerers").

Results: In an ANOVA $(\mathbf{F}(2,815)=398.50, p<.0001)$, significantly lowest MFAST scores were those of healthy controls (mean=1.59, $\mathrm{SD}=\mathbf{2 . 8 0})$, the next significantly higher scores were those of legitimate patients (mean $=4.85$, $\mathrm{SD}=4.22$ ), and the instructed malingerers had significantly highest scores $($ mean=12.34, $\mathrm{SD}=5.71)$.

Discussion: The significant difference between healthy controls and patients shows that inferences from analog validations of the M-FAST are inherently false. Furthermore, data of legitimate patients with severe psychiatric illness suggested that they may face the risk of about $50 \%$ to be falsely classified as malingerers by the M-FAST. Moreover, almost all validations of the M-FAST were done only with "instructed malingerers" (healthy volunteers instructed to feign symptoms). This overestimates the test's capacity to detect real-life malingerers. Montes and Guyton documented that "instructed malingerers" warned to avoid detection score much lower than the unwarned ones (effect size: Cohen's d=3.05). MFAST's capacity for detection of real-life malingerers may be extremely low, in particular those more genuinely motivated to evade detection, well prepared, better educated, and systematically feigning only a few specific symptoms such as depression, pain, and insomnia.

Conclusion: The M-FAST should no longer be used.

Keywords: Malingering, M-FAST, criterion validation, convergent validation, analog validation, meta-analysis.

\section{INTRODUCTION}

The Miller Forensic Assessment of Symptoms Test (MFAST) [1] was developed by Holly A. Miller to identify malingering of mental illness by forensic inmates, but its use has soon been expanded to many other clinical populations without being accurately validated for an "off label use."

The M-FAST has been translated into other languages, including also Armenian [2], Persian [3], and Turkish [4] and is used by thousands of psychologists who appear unaware that the M-FAST is essentially an iatrogenic test, misclassifying too many genuine patients as "malingerers," thus depriving them of therapies and of other legally owed benefits.

The iatrogenic nature of the M-FAST is primarily due to its item pool: it consists of 25 items none of which have the
Submitted : May 26, 2021

Published : June 19, 2021

ISSN: $2593-8339$

DOI: 10.24018 / ejmed.2021.3.3.895

\section{Z. Z. Cernovsky*}

Department of Psychiatry, Western

University, Ontario, Canada.

(e-mail: zcernovs@ uwo.ca)

*Corresponding Author reasonable capacity of properly differentiating true malingerers from genuine psychiatric patients [5]. The items describe mainly legitimate medical symptoms that, in the MFAST, are fallaciously scored as indicative of malingering.

The item content covers 2 major areas:

(1) psychiatric symptoms such as depression or those encountered in acute psychoses (delusions, hallucinations, thought disorder);

(2) symptoms of the post-concussion or whiplash syndrome (e.g., tinnitus [6], formication [7], phantosmia [8], impaired cognitive processing).

Accordingly, high rates of false positives (i.e., of genuine patients falsely branded as malingerers) with the M-FAST may be obtained when testing forensic inmates for feigning mental illness, or while screening injured motorists, injured 
industrial workers, or combat veterans for feigning impairments of physical or mental health.

Damaging evidence against M-FAST comes from reviews of item content of the M-FAST by experienced clinicians, [5] from published rates of false positives found with genuine patients [9] from statistics on the nature of most frequently endorsed M-FAST items by US combat veterans with PTSD [10] and from psychometric studies of scientists in the US Veterans Administration (VA) led by Erika Wolf [11].

In the M-FAST study by VA scientists led by Erika Wolf, the patients' responses to each individual item of the MFAST were compared to results of subsequent clinical interviews [11]. For instance, M-FAST Item 2 ("feeling depressed") was endorsed initially by $67.8 \%$ of veterans with probable PTSD (thus classifying about $2 / 3$ of these veterans as "malingering depression"), but then subsequent clinical interviews determined that only $5.7 \%$ of them might be feigning those symptoms [11]. With respect to the M-FAST's capacity to assess malingering, Wolf's team concluded, that "despite its widespread use, the M-FAST may be insufficient for this purpose and that simply eliminating poorly performing items or conducting follow-up interviews may likewise be inadequate for addressing concerns related to the measure's reliability, validity, and utility in veteran PTSD samples."

Many formal flaws prevent the M-FAST from being an adequately constructed psychological test. For instance, some M-FAST items consist of two parts: the second one extensively alters the logical meaning of the first part. Patients with impaired concentration are prone to misinterpret such items. Numerous misunderstandings, by the patients, of the item text were noted in the VA study by Wolf's team [11]. The misunderstandings might be more frequent in acutely ill psychiatric patients with thought disorder or in patients with post-concussive symptoms, fatigue, or those distracted by persistent pain such as motorists with lingering post-accident symptoms from high impact collisions, or war veterans with post-concussive symptoms from exposure to explosions.

The misunderstandings of M-FAST items by these patients are also promoted by instructions for the test administration in M-FAST manual. The instructions require that the patients are rushed through the test and that patients' requests for clarifications of the meaning of individual items should be answered only evasively, see M-FAST manual [1], page 10: "Some examinees will typically respond to interview items with questions of their own. With few exceptions, the interviewer should respond by saying 'I am interested in your perceptions,' and then repeat the item." And on the same page of M-FAST manual [1]: "It is very important for the interviewer to correctly set the stage from the beginning of the administration. Allowing the examinee to provide no response or to elaborate extensively on his or her symptoms will likely result in a difficult, time-consuming, and nonstandardized administration. Interviewers may believe that it is impolite to interrupt when an examinee begins to elaborate on his or her symptoms or psychological problems during the interview. However, the interviewer must be able to redirect the examinee to respond to each item with minimal or no elaboration".

Each M-FAST item, if endorsed in the alleged direction of malingering, counts one point. The cutoff for classifying the patient as malingering is the score of 6 or more points, as indicated by Holly Miller in her M-FAST manual [1], page 16: "Although the presence of certain psychiatric disorders may result in the endorsement of some items, it is unlikely that an individual with an authentic clinical disorder will obtain an M-FAST Total score of 6 or greater".

Recent M-FAST study examined the seven most frequently endorsed M-FAST items by a sample of $121 \mathrm{US}$ combat veterans with probable PTSD [10]. Since the MFAST cutoff score is 6 or more points, these 7 items were those most instrumental in falsely classifying the group of 121 veterans as only feigning their symptoms. All 7 items represent legitimate medical symptoms fallaciously scored by the M-FAST scoring system as malingering. For instance, the first four of those items most frequently endorsed by veterans are Item 2 ("feeling depressed most of the time," endorsed by $67.8 \%$ of veterans), Item 23 ("feeling that I don't really matter," endorsed by $56.2 \%$ ), Item 20 (formication [7] i.e., "false sensation as if insects move on or under the skin," endorsed by $28.9 \%$ ), and Item 17 (phantosmia [8] i.e., "false sensation of an odor," endorsed by $24.0 \%$ ). The Item 2 and Item 23 denote the depressive mood and discouragement as prevalent among combat veterans with PTSD: these symptoms are not pathognomonic of malingering or exclusive to malingerers. The formication and phantosmia are legitimate neuropsychological symptoms [7], [8] potentially consistent with whiplash injuries or head trauma from repeated explosive blasts or exposure to toxic chemicals [12] in the war zone. Subjective awareness of phantosmia or formication may be more salient when the patient is less distracted by external stimuli, e.g., while attempting to fall asleep (i.e., as specified in the text of M-FAST Item 17 "at times, when you cannot fall asleep").

In her M-FAST manual, to document the "criterion validity" of her test, Holly Miller described a study in which she compared M-FAST scores of students instructed to malinger mental illness with those responding honestly. Since the M-FAST is mainly only a list of legitimate symptoms [5] each of which when reported is scored with one point as indicative of malingering, her procedure differentiates only reporters from non-reporters of symptoms. This method does not verify the capacity of the M-FAST to differentiate malingerers from legitimate patients: both groups could report a similar number of symptoms. Her method (comparing "instructed malingerers" to healthy "honest responders") is known as "analog validation" as it includes no patient group. Recently, it was shown that the analog validation procedure would, very absurdly, "validate" even the famous Beck Depression Inventory, version 2 (BDI-II) [13] as a measure of malingering, thus branding severely depressed (and perhaps suicidal) patients, i.e., the high BDIII scorers, as malingerers [14].

Under the same heading of "criterion validity," Miller reports in her M-FAST manual her 3 studies comparing scores on Rogers's Structured Interview of Reported Symptoms (SIRS) [15] to M-FAST scores. At that time, more than 2 decades ago, the SIRS (an early version of the currently used SIRS-2) was considered the "gold standard" for detection of malingering, with some professionals considering it as almost infallible. However, a recent metaanalysis by Green and Rosenfeld [16] shows that research on 
the SIRS since its initial validation procedures has demonstrated lower specificity rates than those reported in the SIRS manual, i.e., higher rates of misclassifying patients as "malingerers," with lower capacity to differentiate the legitimate patients from malingerers. Above all, calculations of a correlation of a new test (the M-FAST) with another test measuring the same or similar construct can examine only socalled convergent validity, but not the criterion validity of the new test. It is an error to misrepresent such studies as those of "criterion validity." The criterion validity is defined as the capacity of the test to differentiate malingerers from genuine patients.

It is noteworthy in this context that some psychologists, for instance, Graue et al. [17] characterized Miller's M-FAST as "essentially a short form of the SIRS." For this reason, high correlations of the M-FAST and the SIRS can be expected, even if both tests were devoid of any criterion validity. The high correlations fallaciously overestimate M-FAST's criterion validity.

It is also noteworthy that, on the acknowledgement page introducing the 2001 edition of her M-FAST manual, Holly Miller thanked the pioneer in studies of malingering, Richard Rogers, the author of the SIRS and SIRS-2, "for his support and helpful comments" [1]. It is not clear why her M-FAST is promoted as a legitimate psychological test when her MFAST manual so blatantly misrepresents analog validation and convergent validation as "criterion validations".

The 2019 meta-analysis by Detullio et al. [18] examined statistically the validity of M-FAST. However, Detullio's meta-analysis is fatally flawed by the inclusion of Miller's analog validation study as well as of studies of convergent validity, for instance, those that (too precariously) relied on the SIRS scores to "precisely" divide patients into malingerers and legitimate patients: as mentioned, the MFAST is according to some psychologists, considered to be "essentially a short form of the SIRS" [17].

Detullio's meta-analysis focused on the effect size: the procedure did not differentiate between M-FAST scores of healthy persons and those of genuine patients, presumably on the unverified assumption that both groups would obtain similarly low M-FAST scores. Obviously, the genuine patients obtain higher M-FAST scores by endorsing M-FAST items that describe their legitimate medical symptoms [5]. The more symptoms the patient has, the higher the total MFAST score, often exceeding 5 points and thus classifying the patient as a malingerer. In general, the more ill or more injured patients are those most likely to be misclassified as malingerers by the M-FAST [5].

Another methodological flaw of Detullio's meta-analysis is the inclusion of Keyvan's work [4] as one of the relevant studies, presumably accepting it as a legitimate validation of the M-FAST. Keyvan's team [4] divided Turkish forensic psychiatric inmates into malingerers and non-malingerers via procedures that included, among others, MMPI scores on the $\mathrm{F}$ (Infrequency) scale and also on its $\mathrm{F}$ minus $\mathrm{K}$ index. As discussed by various MMPI and MMPI2 experts over several decades, the interpretation of $\mathrm{F}$ and also $\mathrm{F}$ minus $\mathrm{K}$ elevations is complex and difficult. Besides "symptom overreporting," these elevations can, among several other possibilities, result from severe "psychological distress" rather than malingering (Greene [19], pages 110-111), with higher F scores indicating greater psychopathology (Graham [20], page 27), perhaps with thought disorder or are consistent with the "cry for help" of a psychiatrically markedly distressed individual (Lachar [21], pages 17-18). Such persons can also obtain correspondingly high M-FAST scores (many M-FAST items describe legitimate symptoms of psychosis but score them as "malingering"). Hence, statistical correlations of M-FAST scores with MMPI $\mathrm{F}$ and $\mathrm{F}$ minus $\mathrm{K}$ scores reported by Keyvan [19] are an illusory and inappropriate evidence of $\mathrm{M}$ FAST validity.

The present study calculated the meta-analysis of M-FAST scores in a different manner. Included were only studies reporting M-FAST scores on one or more of the following categories of samples: (1) healthy controls, (2) genuine patients, and (3) instructed malingerers. The meta-analysis was to compare scores of these 3 groups. Excluded were studies that relied on dubious methods of dividing patients into a genuine patient's group and malingerers, such as the SIRS scores as a (fallacious) evidence that the M-FAST classified such SIRS derived groups relatively "correctly".

Keyvan's study was also excluded. Excluded were also some other studies listed in Detullio [18] such as doctoral dissertations of Dahlstrom from 2010, by Randolph in 2018, Snyder in 2012, and Waybright in 2010, because they are not easily available to scientists attempting to replicate metaanalytic calculations.

\section{METHOD}

In the present study, as mentioned, the eligible samples of M-FAST scorers were divided into three main groups, see Table I:

(1) those consisting only of normal controls $(\mathrm{N}=231)$,

(2) those consisting only of patients $(\mathrm{N}=305)$, and

(3) those consisting of instructed malingerers or also presumed malingerers $(\mathrm{N}=382)$.

The presumed malingerers included 44 persons identified so by Iranian clinical staff in the study by Ahmadi [3] and also 8 persons identified as malingering by Jackson, Rogers, and Sewell [22] based on their SIRS scores (their 8 persons were then statistically treated as true "malingerers").

\section{RESULTS}

The mean M-FAST scores and SDs of various samples in the three groups are listed in Table I.

The average score was calculated for each of these three groups of samples via the procedure of weighting the averages according to individual sample size. Computational formula for averaging SDs is available in textbooks of statistics such as by Downie and Heath [23].

The last three rows of Table I indicate the mean scores and SDs of the three groups.

A. ANOVA Calculated to Compare Mean Scores of Controls $(N=231)$, Patients $(N=305)$, and Malingerers $(N=382)$

The ANOVA compared the three groups listed in the last three rows of Table I and led to a significant overall $\mathrm{F}$ value: $\mathrm{F}(2,815)=398.50, \mathrm{p}<.0001$. 
Tukey HSD Post-hoc Tests indicated statistically significant differences between all three groups:

Controls vs Patients: Diff $=3.2600,95 \% C I=2.3422$ to 4.1778, $p=0.0000$.

Controls vs Malingerers: Diff $=10.7500,95 \% C I=9.8162$ to 11.6838, $p=0.0000$.

Patients vs Malingerers: Diff $=7.4900,95 \% C I=6.6207$ to 8.3593, $p=0.0000$.

Briefly, the normal controls endorsed less M-FAST items, i.e., less of potentially legitimate medical symptoms, than did the patients. In turn, the patients tended to endorse less of the potentially legitimate medical symptoms than did the malingerers.

TABLE I: M-FAST SAMPLES INCLUDED IN META-ANALYSIS

\begin{tabular}{|c|c|c|}
\hline \multicolumn{3}{|c|}{ M-FAST data (total N=918) } \\
\hline & Mean (SD): & $\mathrm{N}:$ \\
\hline \multicolumn{3}{|l|}{ NORMAL CONTROLS: } \\
\hline Montes and Guyton [24] & $0.82(1.14)$ & 34 \\
\hline Jackson, Rogers, and Sewell [22] & $1.60(2.64)$ & 96 \\
\hline Weiss \& Rosenfeld [9] & $5.3(5.1)$ & 24 \\
\hline Minoudis [25] & $0.7(1.2)$ & 30 \\
\hline Miller [1] & $0.81(1.12)$ & 47 \\
\hline \multicolumn{3}{|l|}{ GENUINE PATIENTS: } \\
\hline Weiss \& Rosenfeld [9] (PTSD) & $9.6(5.8)$ & 19 \\
\hline Ter-Stepanyan et al. [2] & $6.83(4.26)$ & 54 \\
\hline Minoudis [25] & $5.2(5.7)$ & 35 \\
\hline Wolf et al. [11] & $4.21(3.60)$ & 121 \\
\hline Ahmadi et al. [3] & $3.13(1.88)$ & 76 \\
\hline \multicolumn{3}{|l|}{ Instructed MALINGERERS: } \\
\hline Wahl et al. [26] & $10.46(4.25)$ & 50 \\
\hline Jackson, Rogers, and Sewell [22] & $8.58(5.48)$ & 43 \\
\hline Ter-Stepanyan et al. [2] & $12.83(3.95)$ & 54 \\
\hline Miller [1] & $16.00(4.18)$ & 47 \\
\hline Minoudis [25] & $12.8(5.3)$ & 40 \\
\hline \multicolumn{3}{|l|}{$\begin{array}{l}\text { Instructed MALINGERERS, warned to } \\
\text { avoid detection: }\end{array}$} \\
\hline Montes and Guyton [24] & $9.44(3.37)$ & 34 \\
\hline Weiss \& Rosenfeld [9] & $13.2(5.9)$ & 28 \\
\hline \multicolumn{3}{|l|}{$\begin{array}{l}\text { Instructed MALINGERERS, not warned to } \\
\text { avoid detection: }\end{array}$} \\
\hline Montes and Guyton [24] & $21.44(4.43)$ & 34 \\
\hline \multicolumn{3}{|l|}{ Presumed MALINGERERS: } \\
\hline Jackson, Rogers, and Sewell [22] & $10.25(3.65)$ & 8 \\
\hline Ahmadi et al. [3] & $8.28(2.12)$ & 44 \\
\hline \multicolumn{3}{|l|}{ TOTALS: } \\
\hline ALL NORMAL CONTROLS POOLED & $1.59(2.80)$ & 231 \\
\hline ALL GENUINE PATIENTS POOLED & $4.85(4.22)$ & 305 \\
\hline ALL MALINGERERS POOLED & $12.34(5.71)$ & 382 \\
\hline
\end{tabular}

B. ANOVA Comparing Mean Scores of Controls $(N=231)$, Patients $(N=305)$, and of Warned Malingerers $(N=62)$

In some of the studies, the "instructed malingerers" were specifically warned to be cautious to avoid detection. The mean M-FAST score calculated by pooling the group of 34 warned instructed malingerers from the study by Montes and Guyton [24] with 28 warned instructed malingerers from the study by Weiss and Rosenfeld [9] was 11.14 (SD=5.01).

The overall ANOVA in which only these 62 warned (i.e., no unwarned malingerers were included) were compared to the controls and patients, was still significant: $\mathrm{F}(2,595)=159.41, \mathrm{p}<.0001$.

Tukey HSD Post-hoc Tests showed that even these "warned malingerers" still scored significantly higher than both the controls and the patients:

Controls vs Patients: Diff $=3.2600$, 95\%CI=2.4744 to 4.0456, $\mathrm{p}=0.0000$.

Controls vs Warned Malingerers: $\quad$ Diff $=9.5500$, $95 \% \mathrm{CI}=8.2618$ to 10.8382 , $\mathrm{p}=0.0000$.
Patients vs Warned Malingerers: Diff $=6.2900$, $95 \% \mathrm{CI}=5.0353$ to $7.5447, \mathrm{p}=0.0000$.

\section{T-test Comparing Warned $(N=62)$ vs Unwarned Malingerers $(N=34)$}

In the 2014 study by Montes and Guyton [24] on incarcerated inmates, 34 of these inmates received the following instruction for the M-FAST: "I want you to pretend and act as if you have a serious mental illness. Before I start asking you these questions, you can take a moment to think about how you will answer the questions to appear "crazy." $B e$ as believable and convincing as possible. I really want you to convince me that you are "crazy" [24]. They were not warned to avoid detection as malingerers.

This group was compared by Montes and Guyton to another sample, drawn from the same population of incarcerated inmates, of 34 inmates who were warned to feign their symptoms cautiously, to avoid detection of feigning [24]. The t-test comparing warned malingerers (mean score $=9.44, \mathrm{SD}=3.37$ ) with unwarned malingerers (mean score $=21.44, \mathrm{SD}=4.43$ ) was statistically significant $(\mathrm{t}=12.57, \mathrm{df}=66, \mathrm{p}<.001,2$-tailed $)$. The effect size of the underlying difference is high: Cohen's $\mathrm{d}=3.05$ or $\mathrm{r}=.84$.

D. ANOVA Comparing Patients $(N=305)$ to Warned $(N=62)$ and also to Unwarned malingerers $(N=34)$

This overall ANOVA was statistically significant: $\mathrm{F}(2,398)=250.07, \mathrm{p}<.0001$.

Tukey HSD Post-hoc Test showed that all three groups significantly differed from each other:

Patients vs warned malingerers: Diff $=6.2900$, 95\%CI=4.8589 to 7.7211, $p=0.0000$.

Patients vs unwarned malingerers: Diff $=16.5900$, $95 \% \mathrm{CI}=14.7327$ to $18.4473, p=0.0000$.

Warned vs unwarned malingerers: Diff $=10.3000$, $95 \% C I=8.1078$ to $12.4922, p=0.0000$.

Briefly, the patients endorsed less M-FAST items, i.e., potentially legitimate medical symptoms, than did the warned malingerers. In turn, the warned malingerers tended to endorse less of the potentially legitimate medical symptoms than did the unwarned malingerers.

\section{E. Estimate of \% of Patients Scoring at or above 6 Points}

The M-FAST cutoff is 6 or more points. The average score of the 305 patients in this meta-analysis was $4.85(\mathrm{SD}=4.22)$. The 4.85 is thus the peak of the score distribution. The cutoff of 6 is to the right side of the peak, at the distance of less than one SD. The z-score calculated for the position of 6 points was 0.27 , thus providing an estimate that approximately $39.36 \%$ (i.e., about $40 \%$ ) of the sample of 305 patients would have an M-FAST score of 6 points or higher. This is the estimated frequency of false positives. The so called "specificity" of this meta-analytically combined sample (i.e., the proportion of patients correctly classified by the M-FAST as non-malingerers) would be 0.61 .

Noteworthy are the high proportions of genuine patients falsely classified as malingerers in some of these individual samples, see Table II listing estimates based on calculations of $\mathrm{z}$-score for the cutoff of 6 points, using the mean and SD of each sample. 
TABLE II: ESTIMATED PROPORTIONS OF FALSE POSITIVES IN THE INDIVIDUAL PATIENT SAMPLES

\begin{tabular}{cccc}
\multicolumn{4}{c}{ INDIVIDUAL PATIENT SAMPLES } \\
\hline & $\begin{array}{c}\text { Means } \\
\text { (SDs) }\end{array}$ & $\begin{array}{c}\% \text { at or } \\
\text { above 6 }\end{array}$ & Specificity \\
\hline Weiss \& Rosenfeld [9] & $9.6(5.8)$ & $73.2 \%$ & .27 \\
Ter-Stepanyan et al. [2] & $6.83(4.26)$ & $57.5 \%$ & .42 \\
Minoudis [25] & $5.2(5.7)$ & $44.4 \%$ & .56 \\
Wolf et al. [11] & $4.21(3.60)$ & $30.9 \%$ & .69 \\
Ahmadi et al. [3] & $3.13(1.88)$ & $6.3 \%$ & .94 \\
\hline
\end{tabular}

These statistical estimates, while useful, can be inaccurate, leading to overestimates or underestimates. For example, the actual rate of M-FAST's false positives in the study by Weiss \& Rosenfeld for genuine patients with high PTSD was $63.2 \%$, i.e., $10 \%$ lower than the estimated rate derived solely from the mean score and SD.

The highest estimated accuracy rate for the M-FAST was obtained with data provided on PTSD in the Iranian military (Ahmadi's study [3]). However, in the majority of the studies ( 3 out of 5) in Table II, the proportions of false positives with the M-FAST suggest an alarmingly high iatrogenic potential.

\section{DISCUSSION}

The M-FAST was developed and "validated" in a methodologically inept manner, including a reliance on the highly flawed procedure of analog validation, and based on correlations to SIRS scores as a confirmation of "criterion validity." Meta-analytic results of the present study provide strong evidence against the use of analog validation because they show that patients tend to obtain significantly higher MFAST scores than normal controls. This indicates that data from healthy controls cannot be substituted for those of genuine patients in "validation studies" such as that published by Holly Miller.

The results of the present meta-analysis may be interpreted by defenders of the M-FAST as partly supportive to the extent that the lowest scores were obtained by the (presumably healthy) pooled control samples, the next higher scores were by pooled genuine patients, and the highest by pooled instructed or presumed malingerers. However, such approximate ranking is flagrantly unreliable and inadequate, with an undue overlap. For instance, mean M-FAST score of genuine patients in the sample by Weiss and Rosenfeld [9] exceeds mean scores of 3 samples of instructed malingerers or presumed malingerers, see Table I. Such irregularities undermine the criterion validity of the M-FAST, show that the cutoff score of 6 points is unreliable, and indicate that the M-FAST is not an appropriate test to determine malingering in individual cases, i.e., in routine forensic or clinical work.

As explained in the next paragraphs, this meta-analytic study leads to yet other important methodological questions. Above all, the representativeness of the samples of patients and also of "instructed" or presumed malingerers seems highly questionable, to the extent detrimental not only to Detullio's [18] meta-analytic results allegedly supportive of M-FAST diagnostic accuracy, but also to the representativeness of data from the present study.

\section{A. The Representativeness of the Samples of Patients}

In 3 of 5 samples of patients, the key mental health complaint was described as the PTSD. The experiences that trigger PTSD may be horrible, such as exposure to cruelties of the war, or to ethnic violence, or political persecution. However, many patients with PTSD are employable and enjoy an otherwise almost normal quality of life while recovering from their symptoms.

Only in 2 of these 5 studies were the persons very severely ill psychiatric patients. In the Armenian study by TerStepanyan [2], the patients had an ICD 10 diagnosis of severe mental illness, mostly schizophrenia or the bipolar disorder: they were mostly inpatients in state psychiatric hospitals $(\mathrm{N}=42)$ or outpatients of a mental health clinic $(\mathrm{N}=12)$. In Minoudis [25] doctoral dissertation, these persons "were psychiatric inpatients recruited from acute inpatient mental health units" in London, UK, and had a "current diagnosis of severe mental illness (e.g., schizophrenia or bipolar affective disorder)." The data in Table II suggest that the diagnostic probability of M-FAST misclassifying such genuine patients with severe mental illness as malingerers is about $50 \%$ (see the mean score of the patient sample of Minoudis and the one of Ter-Stepanyan in Table II). Briefly, the data indicate that the M-FAST should not be used on such patient groups at all.

Severe mental illness exerts a terrible burden on the patient and the family. Falsely denying therapies and other lawfully owed benefits, no matter how well the M-FAST psychologist is paid for such services by an insurance company or another employer, can be interpreted by other professionals or also by the patient's family members as a sign of the M-FAST psychologist's sociopathy.

Mental patients with thought disorder, delusions, and hallucinations are prone to earn many M-FAST points towards malingering because a large part of its items deal with hallucinations, delusions, and strained logics. Persons who develop such acute symptoms while incarcerated are especially vulnerable to the charlatanism inherent in M-FAST assessments of malingering. As discussed elsewhere [5]: "the M-FAST items list many legitimate psychiatric symptoms that are (rather perplexingly) scored as indicators of malingering. A few examples are as follows: auditory hallucinations ("voices") associated with autonomic signs of anxiety (Item 18) or with fear of leaving the room or home during such episodes (Item 22), hallucinations lasting for days (Item 6), and olfactory hallucinations (phantosmia) (Item 17), adverse changes of mood while suspecting to be plotted against (Item 3), a belief to have special powers with respect to sensory perception (Item 13), and delusional parasitosis (Item 20). An M-FAST item refers to "feeling depressed most of the time" (Item 2) and is also scored as indicator of malingering" [5].

The M-FAST was originally developed for forensic assessments of malingering mental illness. However, of much current concern is also its frequent "off label" use on injured patients with the polytraumatic symptom patterns (pain, depression, post-concussion and whiplash syndrome, anxiety), e.g., on persons injured in high impact car accidents, or injured industrial workers, veterans with lingering symptoms from battlefield injuries, etc. Such clinical populations with physical and neuropsychological injuries are adequately represented neither by samples in the fallacious meta-analysis of Detullio [18], and unfortunately, nor in the meta-analytically pooled samples in the present study.

Readers with interest in the abuse of US veterans by MFAST psychologists should consult Table I in a recent 
publication [10] summarizing M-FAST items most frequently endorsed by veterans with probable diagnosis of PTSD: as already explained, the content of those items seems to describe potentially legitimate medical symptoms of veterans, but the items are scored by the M-FAST as indicating malingering.

An extensive discussion of M-FAST items listing symptoms of these clinical groups that the M-FAST fallaciously scores as indicative of malingering was provided elsewhere [5]. With respect to symptoms encountered in injured motorists, examples of inappropriate item content (inadequate to differentiate legitimate patients from malingerers) in the M-FAST include: "depressed feelings (Item 2), tinnitus triggered or exacerbated over the duration of stressful interview (Item 25), intense nightmares that occur concurrently with weight loss (Item 12), neurological symptom of formication (Item 20), phantosmia (Item 17), and fluctuation of symptoms as if someone is "turning them on and off ..." (Item 14)'[5].

\section{B. MRIs versus M-FAST Assessments}

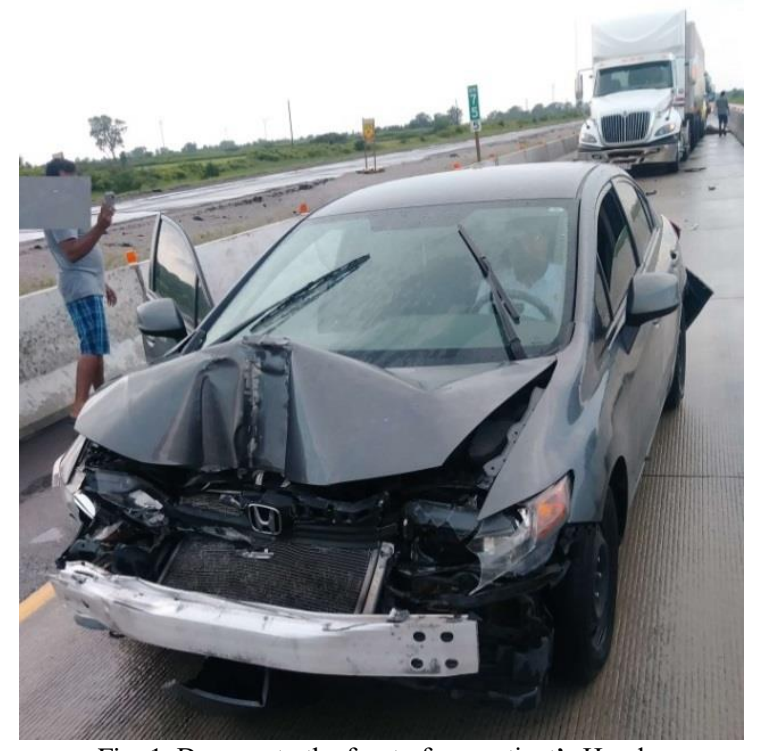

Fig. 1. Damage to the front of our patient's Honda.

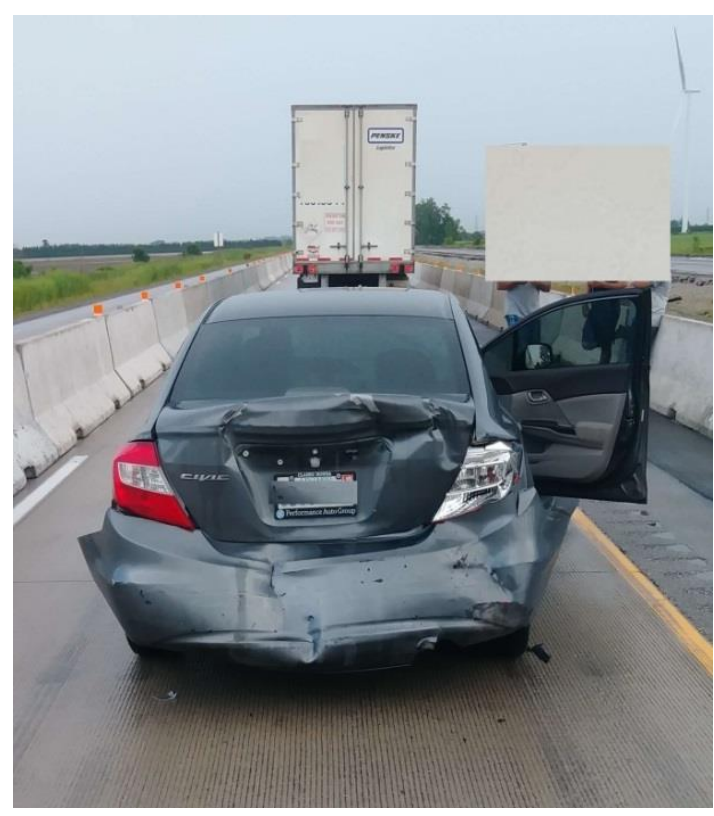

Fig. 2. Damage to the rear of our patient's Honda.
MRIs of spinal injuries have been used with increasing frequency over the recent years for accurate assessments of cervical whiplash (WAD-C), thoracic whiplash (WAD-T), and lumbosacral whiplash (WAD-LS) [27]. There is a corresponding increase in well documented false M-FAST assessment reports by psychologists which declare the patients as malingering pain and other whiplash symptoms even when their MRIs have adequately documented impinged nerves and damage to spinal discs and other tissues. Frequent discrepancy between the MRIs and such false psychological reports may eventually attract negative attention from physicians, personal injury lawyers, and governmental agencies such as those regulating insurance re-imbursements to prevent fraud. This is likely to have an adverse impact on the credibility of psychology as a profession allegedly based on scientific evidence, but, in fact, too often relying on fallacious statistical conclusions such as Detullio's.

A psychologist using the M-FAST may receive as much as $\$ 10,000$ per assessment report. The folly of some of these assessments can be illustrated by the following example, described in more detail elsewhere [5]: a 57 year old gentleman with a master's of science degree in chemistry drove "his Honda through a construction area on an expressway ..... the traffic ahead slowed down, and his car was rear-ended by a transport truck. The impact propelled his Honda into another transport truck ahead. The photographs show very extensive damage both to the front and to the back of his Honda: the car was later deemed not worthy of repair ..... "[5] The patient briefly lost consciousness and "he recalls subsequently feeling disoriented, confused, stunned, dazed, and dizzy (presumptive signs of cerebral concussion)" [5], [28]. An ambulance transported this patient "to a hospital where he underwent a physical examination, $X$-rays, and received analgesic medication. The MRI showed compression fractures of lumbar vertebrae" [5]. The patient reported persistent pain, pain related insomnia, and numerous unremitting postconcussive and whiplash symptoms.

The insurance contracted psychologist interpreted this patient's M-FAST score as suggesting "a clear potential for exaggerating or feigning psychological distress" and declared the patient as able to resume his employment tasks (teaching highschool students) after this motor vehicle accident (MVA). The insurance contracted psychologist concluded in his report: "In my opinion, there was no reliable evidence suggesting MVA-related psychological symptoms would result in an inability to perform essential and nonessential tasks of pre-MVA employment."

In the aftermath of receiving the derogatory psychological report implying that he is feigning his symptoms, the patient eventually experienced a cardiac crisis and underwent a cardiac surgery.

Subjectively reported psychological symptoms such as the intensity and subjective quality of pain, and pain related insomnia, various post-concussive and whiplash symptoms, PTSD, post-accident depression and anxiety are measured by psychological scales [13], [29]-[35] that should be evaluated for their correlations to spinal MRIs of such post-MVA patients.

When denying the validity of self-reports by the patient, the assumption that a secondary gain is present is often 
mentioned in reports by insurance contracted psychologists: the patient's application for insurance benefits is paradoxically presented as evidence of secondary gain that disqualifies the injured patient from entitlement for therapies and other insurance benefits. Such arguments incongruously undermine the legal purpose of mandatory car insurance.

In general, the more ill or more injured patients, those with more symptoms, are more likely to be classified as malingerers by the M-FAST.

\section{C. “Instructed Malingerers" versus True Malingerers}

As already explained, if the purported "validation" of the M-FAST only compares a sample of healthy persons instructed to respond honestly to a sample of instructed malingerers, the instructed malingerers obviously report more symptoms on the M-FAST than healthy individuals. Since the alleged purpose of M-FAST is to differentiate malingerers from legitimate patients, scientific validation studies must compare these 2 groups. The validation must include legitimate patients. Resorting instead to comparisons of honestly responding healthy control samples to samples of instructed malingerers (i.e., using analog validations) is methodological quackery. There is also a major methodological problem with the use of instructed malingerers in lieu of real-life malingerers. Almost all validations of the M-FAST have been done only with "instructed malingerers" (healthy volunteers instructed to feign symptoms).

As explained in the Result section, the excellent experimental work of Montes and Guyton [24] compared unwarned instructed malingerers to their counterparts warned to proceed cautiously to avoid detection. The mean score of the unwarned was about twice higher than the one of warned instructed malingerers, see Table I.

The mean number of years of education of these instructed malingerers was only 10.4 in the warned and 10.0 in the unwarned group: they were all incarcerated inmates. However, even groups of college or university students recruited to be "instructed malingerers" tend to obtain high scores such as 16 points in Miller's study [1], perhaps due to lack of genuine and adequately intensive incentive or of serious concern to indeed avoid detection. Furthermore, the M-FAST consists only of 25 items: these can be conceived of as a "menu" from which the "instructed malingerer" urged to feign mental health difficulties is choosing some symptoms to feign. This raises the question to what extent so called "instructed malingerers" can adequately represent, or experimentally substitute for, real malingerers who are highly motivated to indeed avoid detection, devote more time to decide which symptoms to feign, and might cautiously limit themselves to reporting very few symptoms, not necessarily those described in M-FAST items. For example, those feigning injuries from a motor vehicle accident might report only pain, insomnia, and perhaps also some depression, as they are not adequately familiar with the full spectrum of potential medical symptoms associated with high impact car accidents such as post-concussive and whiplash symptoms (e.g., tinnitus, formication, phantosmia, cognitive impairment). Since there is only one M-FAST item clearly descriptive of depression, and none assessing insomnia or pain per se, such true malingerers are likely to remain undetected as they never reach the cutoff of 6 points. This means that the capacity of M-FAST to detect such true malingerers (as opposed to "instructed malingerers") is so minimal, that the test is useless.

Similarly, true malingerers of mental illness might be aware from books, films, or internet sources that symptoms of severe psychiatric illness such as hallucination or delusions tend to have highly individual content for each person (for instance, not every psychotic patient believes to be "a reincarnation of Napoleon" or of "Rene Descartes"). Their prior acquired knowledge, combined with lack of thought disorder, would prompt these real-life malingerers to avoid some of the traps of the M-FAST and thus the detection.

The statistics known as "test sensitivity," in the case of MFAST, is its capacity to detect true malingerers. Briefly, there are good reasons to assume that M-FAST's "sensitivity" statistics based on studies of instructed malingerers are crude overestimates that mislead the public about the actual capacity of M-FAST to detect real-life malingerers. As already explained, true malingerers might cautiously limit themselves to reporting very few symptoms, not necessarily those described in M-FAST items. While the M-FAST may detect careless, poorly prepared, and impulsive "instructed" malingerers who lack in adequate motivation to evade detection, and perhaps also have low educational level (such as in the study of unwarned instructed malingerers by Montes and Guyton [24]), the M-FAST's ability to detect more careful, highly motivated, well prepared, and well educated malingerers feigning only a few specific symptoms may be extremely poor, almost non-existent. If this is true, then MFAST psychologists primarily falsely classify legitimate patients as malingerers, depriving them of therapies and other insurance benefits. Their claim that M-FAST can detect reallife malingerers may be grossly exaggerated.

\section{Epidemic of the M-FAST}

M-FAST has been used with increased frequency in the last 10 years, as evidenced also by its translations into other languages such as Turkish [4], Persian [3], or Armenian [2]. The evidence damaging to M-FAST's validity is also growing. At the same time, studies similar to the one by Detullio [18] ultimately "muddy the waters" to obstruct the public awareness of abuse of legitimate patients by M-FAST psychologists. Since the use of M-FAST has been considered as "common and accepted practice," similarly to bloodletting in medicine more than 2 centuries ago, there is still much resistance among M-FAST users to acknowledge the inherently iatrogenic potential of this test. Unfortunately, some of these entrenched users are well respected members of editorial boards of major psychological journals, or prominent figures in national psychological associations, or university professors: some of them are erroneously promoted as special experts in the detection of malingering.

\section{CONCLUSIONS}

The present meta-analysis indicates that M-FAST scores of genuine patients are significantly higher than those of healthy controls: this fact alone disqualifies the use of analog validations. Methodological considerations also suggest that 
so called "instructed malingerers," as used in the purported validation studies of the M-FAST, are a too inadequate substitute for true malingerers many of whom can be far more motivated, more careful, well prepared, and well educated and avoid detection more successfully: the "sensitivity" statistics published by proponents of M-FAST may be crude overestimates that mislead the public. The "specificity" of the M-FAST is too substandard, as shown by high rates of false positives in methodologically relatively adequate studies. The use of M-FAST should be discontinued immediately.

\section{ACKNOWLEDGMENT}

The author thanks Abe Cernovsky, B. A., for his editorial assistance on this manuscript.

\section{REFERENCES}

[1] Miller HA. M-FAST. Miller Forensic Assessment of Symptoms Test. Professional Manual. Lutz, FL: PAR Inc., 2001.

[2] Ter-Stepanayn A, Azizian A, Gasparyan K, Ghazaryan G, \& English P. Assessing Initial Psychometric Properties of the Armenian Version of the Miller Forensic Assessment of Symptoms Test (M-FAST) with Psychiatric Patients and Simulator Participants. Journal of Forensic Psychology Research and Practice. 2021, DOI: 10.1080/24732850.2021.1879209.

[3] Ahmadi K, Lashani Z, Afzali MH, Tavalaie SA, \& Mirzaee J. Malingering and PTSD: Detecting malingering and war related PTSD by Miller Forensic Assessment of Symptoms Test (M-FAST). BMC Psychiatry. 2013; 13: 154. http://dx.doi.org/10.1186/1471-244X-13154.

[4] Keyvan A, Ger MC, Ertürk SG, \& Türkcan A. The validity and reliability of the Turkish version of the Miller Forensic Assessment of Symptoms Test (M-FAST). Nöro Psikiyatri Arsivi (Archives of Neuropsychiatry).2015;52:296-302. http://dx.doi.org/10.5152/npa.2015.7587.

[5] Cernovsky Z, Mann S, Diamond DM, Persad E, Oyewumi LK, Velamoor V, Mendonça J, Woodbury-Fariña MA, and Husni M. Critical Review of the Content Validity of Miller Forensic Assessment of Symptoms Test (M-FAST). Archives of Psychiatry and Behavioral Sciences. 2020;3(2):16-29.

[6] Cernovsky ZZ, Mann SC, and Velamoor VR. Neuropsychological Correlates and Frequency of Tinnitus in Patients Injured in Motor Vehicle Accidents. European Journal of Medical and Health Sciences. 2021;3(2):103-106. doi: 10.24018/ejmed.2021.3.2.771.

[7] Cernovsky ZZ, Velamoor VR, Mann SC, Oyewumi LK, Mendonça JD, and Litman LC. Frequency of Formication Symptoms in Injured Motorists and in Normal Controls. European Journal of Medical and Health Sciences. 2021;3(2):48-52.

[8] Doty RL, Yousem DM, Pham LT, Kreshak AA, Geckle R, Lee WW. Olfactory Dysfunction in Patients with Head Trauma. Archives of Neurology. 1997 54(9):1131-40.

[9] Weiss RA, \& Rosenfeld B. Identifying feigning in trauma exposed African immigrants. Psychological Assessment. 2017; 29: 881-889. http://dx.doi.org/10.1037/pas0000381.

[10] Cernovsky ZZ, Mann SC, and Velamoor VR. Clinical profile of US combat veterans with PTSD on M-FAST items. European Journal of Clinical Medicine. In press.

[11] Wolf E, Ellickson-Larew S, Guetta RE, Escarfulleri S, Ryabchenko K, and Miller MW. Psychometric performance of the Miller Forensic Assessment of Symptoms Test (M-FAST) in veteran PTSD assessment. Psychological Injury and Law. Published online April 15, 2020. https://doi.org/10.1007/s12207-020-09373-y.

[12] Thawani S, Wang B, Shao Y, Reibman J and Marmor M. Time to Onset of Paresthesia Among Community Members Exposed to the World Trade Center Disaster. International Journal of Environmental Research and Public Health. 2019, 16, 1429.

[13] Beck AT, Steer RA, Brown GK. Manual for Beck Depression Inventory-II. San Antonio: TX: Psychological Corporation, 1996.

[14] Cernovsky Z, Mann S, Diamond DM, Mendonça J, Tenenbaum S, Persad E, Velamoor V, Woodbury-Fariña MA, Husni M, Gutierrez J. Irremediably Flawed Nature of Analog Validation Methodology of Malingering Tests. Archives of Psychiatry and Behavioral Sciences. 2020; 3(2): 39-45.
[15] Rogers R, Bagby RM, \& Dickens SE. SIRS: Structured Interview of Reported Symptoms: Professional Manual. Odessa, FL.: Psychological Assessment Resources, 1992.

[16] Green D, and Rosenfeld B. Evaluating the Gold Standard: A Review and Meta-Analysis of the Structured Interview of Reported Symptoms. Psychological Assessment. 2011, 23(1), 95-107.

[17] Graue LO, Berry DTR, Clark JA, Sollman MJ, Cardi M, Hopkins J, \& Dellynda Werline D. Identification of Feigned Mental Retardation Using the New Generation of Malingering Detection Instruments: Preliminary Findings. The Clinical Neuropsychologist. 2007;21(6):929-942. DOI: 10.1080/13854040600932137.

[18] Detullio D, Messer SC, Kennedy TD, and Millen DH. A Meta-Analysis of the Miller Forensic Assessment of Symptoms Test (M-FAST) Psychological Assessment. 2019;31(11):1319-1328. http://dx.doi.org/10.1037/pas0000753.

[19] Greene RL. The MMPI-2 / MMPI: An Interpretive Manual. Nordham Heights, MA, USA: Simon and Schuster, 1991.

[20] Graham JR. MMPI2. Assessing Personality and Psychopathology. $5^{\text {th }}$ Edition. New York: Oxford University Press, 2012.

[21] Lachar D. The MMPI: Clinical Assessments and Automated Interpretation. Los Angeles, CA: Western Psychological Services, 1974.

[22] Jackson RL, Rogers R, \& Sewell KW. Forensic applications of the Miller Forensic Assessment of Symptoms Test (M-FAST):

Screening for feigned disorders in competency to stand trial evaluations. Law and Human Behavior. 2005; 29: 199-210. http://dx.doi.org/10.1007/s10979-005-2193-5.

[23] Downie NM, and Heath RW. Basic Statistical Methods. $5^{\text {th }}$ Edition. Harper \& Row: New York, 1983.

[24] Montes O, \& Guyton MR. Performance of Hispanic inmates on the Spanish Miller Forensic Assessment of Symptoms Test (M-FAST). Law and Human Behavior. 2014; 38: 428-438. http://dx.doi.org/10.1037/lhb0000074.

[25] Minoudis PG. Malingering of cognitive symptoms. Doctoral thesis, University College London, UK, 2007. Obtained from: http://discovery.ucl.ac.uk/1444848/.

[26] Wahl KD, Vitacco MJ, Panza NR, \& Hyde TF. Utility of the Inventory of Legal Knowledge in detecting feigning. Journal of Forensic

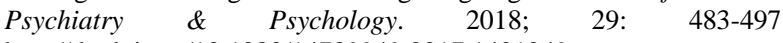
http://dx.doi.org/10.1080/14789949.2017.1421249.

[27] Cernovsky ZZ, Mann SC, Velamoor VR, and Oyewumi LK. The Need for Three Separate Parallel WAD Ratings of Whiplash Injuries to Cervical, Lumbosacral, and Thoracic Spine in Clinical Assessments of Injured Motorists. European Journal of Medical and Health Sciences. 2021; 3(1): 154-159. doi: 10.24018/ejmed.2021.3.1.699.

[28] Cernovsky Z, Istasy P, Bureau Y, \& Chiu S. Scale for retrospective assessment of immediate concussion symptoms. Mental Illness. 2018; 10(2): 70-71.

[29] Cleeland CS. The Brief Pain Inventory - User Guide. Houston, TX: The University of Texas - M. D. Anderson Cancer Center, 2009.

[30] Morin CM, Belleville G, Bélanger L, and Ivers H. The insomnia severity index: psychometric indicators to detect insomnia cases and evaluate treatment response. Sleep. 2011; 34:601-608

[31] King NS, Crawford S, Wenden FJ, Moss NEG, Wade DT. The Rivermead Post Concussion Symptoms Questionnaire: a measure of symptoms commonly experienced after head injury and its reliability. Journal of Neurology. 1995; 242: 587-592.

[32] Cernovsky ZZ, Mann SC, Velamoor V, Oyewumi LK, Diamond DM, Litman LC. Validation of the Rivermead Post-Concussion Symptoms Questionnaire (RPQ) on Patients Injured in High Impact Car Accidents. Archives of Psychiatry and Behavioral Sciences. 2021;4(1):14-22. doi.org/10.22259/2638-5201.0401003.

[33] Cernovsky ZZ, Litman LC, Mann SC, Oyewumi LK, Bureau Y, Mendonça JD, Diamond DM, and Raheb H. Validation of the Subjective Neuropsychological Symptoms Scale (SNPSS) in Injured Motorists. Archives of Psychiatry and Behavioral Sciences. 2021;4(1):6-13.

[34] Weathers FW, Litz BT, Keane TM, Palmieri PA, Marx BP, \& Schnurr PP. The PTSD Checklist for DSM-5 (PCL-5). The National Center for PTSD, US Department of Veterans Affairs, Washington, DC, 2013. www.ptsd.va.gov.

[35] Beck AT, Epstein N, Brown G, \& Steer RA. An inventory for measuring clinical anxiety: Psychometric properties. Journal of Consulting and Clinical Psychology. 1988;56: 893-897.

Zack Z. Cernovsky is the professor of psychiatry in the medical school of Western University, London, Ontario, Canada. Dr. Cernovsky has published more than 200 scientific articles in the field of psychiatry and medical psychology, and also chapters in university textbooks. 